\title{
Raman Tweezers for Tire and Road Wear Micro- and Nanoparticles Analysis
}

Raymond Gillibert, ${ }^{1, *}$ Alessandro Magazzù, ${ }^{1}$ Agnese Callegari, ${ }^{2}$ David Bronte-Ciriza, ${ }^{1}$ Antonino Foti, ${ }^{1}$ Maria Grazia Donato, ${ }^{1}$ Onofrio M. Maragò, ${ }^{1}$ Giovanni Volpe, ${ }^{2}$ Marc Lamy de La Chapelle, ${ }^{3}$ Fabienne Lagarde, ${ }^{3}$ and Pietro G. Gucciardi ${ }^{1, \dagger}$

${ }^{1}$ CNR - IPCF, Istituto per i Processi Chimico-Fisici, Viale F. Stagno D’Alcontres 27, I- 98158 Messina, Italy

2 Department of Physics, University of Gothenburg, 41296 Gothenburg, Sweden

${ }^{3}$ Institut des Molécules et Matériaux du Mans, UMR 6283 CNRS, Le Mans Université, Le Mans, France

Keywords: microplastics, nanoplastics, Tire and Road Wear Particles, Raman spectroscopy, optical tweezers.

\footnotetext{
* Present address: Dipartimento di Fisica, Università di Roma La Sapienza, Piazzale A. Moro 2, I-00185 Rome, Italy

${ }^{\dagger}$ Corresponding author. Email address: gucciardi@ipcf.cnr.it (P. G. Gucciardi)
} 
Abstract. Tire and Road Wear Particles (TRWP) are non-exhaust particulate matter generated by road transport means during the mechanical abrasion of tires, brakes and roads. TRWP accumulate on the roadsides and are transported into the aquatic ecosystem during stormwater runoffs. Due to their size (sub-millimetric) and rubber content (elastomers), TRWP are considered microplastics (MPs). While the amount of the MPs polluting the water ecosystem with sizes from $\sim 5 \mu \mathrm{m}$ to more than $100 \mu \mathrm{m}$ is known, the fraction of smaller particles is unknown due to the technological gap in the detection and analysis of $<5 \mu \mathrm{m}$ MPs. Here we show that Raman Tweezers, a combination of optical tweezers and Raman spectroscopy, can be used to trap and chemically analyze individual TWRPs in a liquid environment, down to the sub-micrometric scale. Using tire particles mechanically grinded from aged car tires in water solutions, we show that it is possible to optically trap individual sub-micron particles, in a so-called 2D trapping configuration, and acquire their Raman spectrum in few tens of seconds. The analysis is then extended to samples collected from a brake test platform, where we highlight the presence of sub-micrometric agglomerates of rubber and brake debris, thanks to the presence of additional spectral features other than carbon. Our results show the potential of Raman Tweezers in environmental pollution analysis and highlight the formation of nanosized TRWP during wear. 


\section{Introduction}

The emission of traffic-related particulate matter (PM) is growing in an uncontrolled manner, causing strong health and environmental concerns on the global scale. ${ }^{1,2}$ While the transition to electric-motor transportation is expected to cut the PM exhaust emission in the next ten years, this is not the case for non-exhaust PM, namely tire-, brake-, and road-wear particles, ${ }^{3,4}$ generally called Tire and Road Wear Particles (TRWPs). Tires particles (TPs) emission in Europe (2014) and US (2010) is estimated in $\sim 10^{6}$ tons per year. ${ }^{4,5}$ PM10 brake wear particles (BWPs), i.e. smaller or equal to $10 \mu \mathrm{m}$, are estimated to reach $80 \mathrm{mg}$ per $\mathrm{km}$ per vehicle. ${ }^{3}$ Furthermore, the demand increase for car tires, ${ }^{6}$ associated with a market trend of heavier vehicles and of electric cars with higher instantaneous torques, is expected to yield larger quantities of TRWPs in the near future.

TWRPs are often transported to the fresh aquatic system via road runoff, and from there to estuaries and oceans. ${ }^{7,8}$ About $20 \%$ of the TPs is known to be released to surface waters. ${ }^{8}$ Due to their size distribution and chemical composition, TWRPs may cause adverse effects on ecosystems and on human health. For example, evidence of toxicity has been found in daphnia magna; ${ }^{9,10}$ air-born tire dust is a probable cause of increased allergy in the general population; ${ }^{11}$ and heavy metals released by TWRPs are known to be harmful to the environment. ${ }^{12-14}$

Knowledge of the TRWP transfer pathways in the environment from roads to the acquatic system is currently incomplete, especially for the smallest particles. To address this knowledge gap and meet the challenges posed by the growing TRWPs pollution, the European TWRP Platform is trying to develop an integrated knowledge of the fate of TRWPs "from road to ocean" and to highlight technological gaps for the study of micro- and nanoscale TRWPs. ${ }^{15}$

TRWPs are generated by the rolling shear of the tires on the road surface and by the friction of the brake pads on the disks. ${ }^{3,4}$ The heating and rubbing of tires during the interaction with the road pavement leads to release of rubber particles incorporating road surface material, generating elongated particles ${ }^{16}$ containing a mix of rubber, dust, asphalt, and brake dust. ${ }^{17,18,19,20}$ Brake wear leads to irregularly shaped and pointy particles that originate from the abrasion of pads, disks and 
calipers. ${ }^{20}$ From the chemical point of view, tires are mostly made of natural and synthetic rubbers ( $\sim 50 \%$ of the mass), typically styrene-butadiene. This elastomer is armed with organic fibers and steel cables, and is charged with carbon, silica and chalk powders. Softeners, such as oils and resins, are used to improve the grip. Zinc oxide and sulfur are used as catalyzer for the vulcanization. ${ }^{20}$ Other additives (5-10\% of the mass), such as cadmium or polyaromatic hydrocarbons (PAH), ${ }^{9,21}$ are also commonly used in the tires as softeners and fillers. ${ }^{12,18}$ The chemical composition of brake wear particles reflect the components of the brake linings: phenol-formaldehyde resins (from the binders); metals such as copper and steel, ceramics and Kevlar (from the reinforcing fibres); inorganic compounds, silicates, stone and metal powders (from the fillers); graphite, carbon black, rubber, antimony trisulfide (from the lubricants); metal oxides quartz and zircon (from the abrasives). ${ }^{3}$ Due to their polymeric content, TPs are typically classified as microplastics (MPs). Given the size range of TPs, these are expected to be a major contributors to micro- and nanoplastics pollution in the near future. ${ }^{4,8}$ Studies indicate TPs as 20 to $60 \%$ of the total MPs delivered to the environment, ${ }^{22,23}$ and no studies have addressed the issue of nanoplastics pollution from TPs, yet.

Wagner et al. classified tire particles based on their size as coarse (PM10, size $<10 \mu \mathrm{m})$, fine (PM2.5, size $<2.5 \mu \mathrm{m})$ and ultrafine (PM0.1, size $<0.1 \mu \mathrm{m}) .{ }^{4}$ Ultrafine TPs $(1 \mathrm{~nm}$ to hundreds of $\mathrm{nm})$ are have been observed in road simulators and seem to originate from organic constituents. Coarse and fine particles $(20 \mu \mathrm{m}$ to $400 \mathrm{~nm}$ ) are found on-road. Particles with a broader size distribution (1 to $200 \mu \mathrm{m}$ ) accumulate in road run-off. Notably, fine particles (PM 2.5) also contribute to the dust air pollution. For what concerns BWPs, ca. $50 \%$ of them are constituted by particulate smaller than 25 $\mu \mathrm{m}$, and almost $40 \%$ is emitted as airborne PM10. ${ }^{3}$

Technological gaps are present in the sampling, handling, storage, extraction and analysis phases of nanoplastics and no standardization exists. ${ }^{24}$ Analytical procedures to establish TWRPs in environmental samples targets at the determination of rubber and additives (metals such as Zn, or S and other organic compounds $)^{25}$ as a distinctive marker for TPs, and of carbonaceous materials and metals (Fe, $\mathrm{Cu}, \mathrm{Zn}$ and $\mathrm{Pb}$, with Fe content reaching $60 \%)^{3}$ in BWPs. To date, SEM imaging with 
elemental analysis (energy dispersive X-Ray spectroscopy, EDXS) and Pyrolysis combined with Gas Chromatography and Mass Spectroscopy (py-GC-MS) are the two election analytical techniques for such purposes. SEM is capable of imaging particles of all length scales from PM0.1 to hundreds of microns. The EDXS information is exploited to identify specific TPs markers, such as $\mathrm{S}$ and $\mathrm{Zn}$, in TWRP dust. ${ }^{18}$ The EDXS signature is not $100 \%$ selective of TPs, however. Zn, for example, may be present also in BWPs and road paint. Py-GC-MS, can be used to identify styrene, butadiene or vinylcyclohexene as markers of rubber in TPs. ${ }^{26}$ The combination of SEM/EDXS with time-of-flight secondary ion mass spectrometry provides elemental information on the rubber component in particles of some tens of microns. ${ }^{27}$ The determination of TPs by Py-GC-MS via the presence of PAHs and aliphatic compounds is more difficult, due to their presence also in asphalt and combustion products. Finally, Py-GC-MS is destructive and is applied to the entire particulate sample, in batches of 10 to $100 \mu \mathrm{g}$, thus preventing analysis at the single particle level.

Raman spectroscopy ${ }^{28,29}$ is a vibrational spectroscopy technique allowing one to chemical identify materials based on the unique energy spectrum of the atomic nuclei vibrations in molecular compounds. It is a non-invasive and non-destructive optical technique enabling analysis and imaging of the materials composition, structural, electronic and chemical properties of micro and nanoparticles on spatial scales generally limited by diffraction to $\sim 250 \mathrm{~nm}$ in the visible range, but that can be pushed to the nanometer and even the sub-nanometer scale using near-field optical microscopy techniques. ${ }^{30-33}$ Raman can provide information on the presence of carbonaceous species (carbon black, graphitic particles), minerals and metal oxides. The Raman fingerprint of carbon, considered by itself, may not be specific to the origin the particle (e.g. car tire, exhaust, lubricant, ash, ...). Additional spectral information of the particle's constituents can, however, help in assessing its species.

The field of nano-pollution, i.e. the environmental pollution caused by nanoparticles and, in particular, nanoplastics is still in its infancy and will need relevant advancements of knowledge, mainly due to the lack of analytical techniques validated by the scientific community, capable to work 
at such length scales. ${ }^{19,34-37}$ Optical tweezers (OT) ${ }^{38}$ exploit tightly focused laser beams to trap and manipulate micro- and nanoparticles in liquid. ${ }^{39}$ The integration of OT with Raman spectroscopy leads to Raman Tweezers (RT), a powerful tool with unique capabilities for the analysis of micro and nanostructures dispersed in liquid, at the single particle level, ${ }^{40,41}$ such as carbon nanotubes and graphene..$^{39,42-45}$ RTs have been shown to be a promising technology in the field of micro- and nanoplastics pollution in aquatic environments, ${ }^{46-48}$ thanks to their capability of single particle analysis in liquid media on timescales going from seconds to few minutes.

In this article, we show that RTs can be applied to TRWPs analysis to obtain chemical identification of micro- and sub-micron particles of artificially fragmented car tires and of environmental samples extracted from a brake testing platform. We first study the experimental configurations that allows one to stably trap and analyze TRWPs, in spite of the large optical pushing effect due to the light absorption, typical of this particles. To show the analytical capabilities of RT, we display Raman spectra of TPs of abandoned tires mechanically grinded and dispersed in water (both distilled and seawater). Micro- and nano- particles in the range of $500-850 \mathrm{~nm}$ are found in the solution, which can be trapped and analyzed in times of few tens of seconds. Finally, we investigate environmental samples extracted from a brake testing platform. Thanks to the presence of spectral features attributable to dust or magnetite, in addition to the Raman bands of carbon, we show the possibility to distinguish TPs from BWPs on particles featuring micron and sub-micrometric dimensions.

\section{Materials and Methods}

Car tire particles. Micron- and sub-micron particles were extracted from an old discarded car tire. They were prepared by scrubbing the rubber fragment against a wet grinding stone (Norton, alundum whetstone for quick sharpening of tools). After each scrubbing cycle, the stone was rinsed repeatedly with $5 \mathrm{~mL}$ of distilled water to collect and disperse the TPs. Dimensional analysis was carried out by 
optical microscopy on a dried sample drop. A second sample was made by dispersing the grinded TPs in seawater taken from the beach of Torre Faro (Messina, Italy).

TWRPs from a brake testing platform. TWRPs were extracted from a brake test platform after locally washing its surface with distilled water. To do so (Supplementary Figure S1), we cast $10 \mathrm{~mL}$ of distilled water on the platform surface and gently mix. After few minutes, a pipette is used to collect the liquid and decant it into a glass vial and immediately sealed in order to avoid contamination from airborne particles.

Raman Tweezers apparatus. The RT setup, shown in Figure $1,{ }^{46}$ is homemade and uses a single laser beam (He-Ne, wavelength $\lambda=633 \mathrm{~nm}$ ) to both optically trap the particles and excite them for Raman analysis. The laser beam is expanded to overfill the entrance pupil of the microscope objective (100X, oil immersion, numerical aperture $N A=1.3$ ), so to get a diffraction limited spot. The resulting spot will be an ellipsoid whose transversal semiaxis is $\Delta r_{\perp}=0.61 \lambda / N A \sim 300 \mathrm{~nm}$ and longitudinal semiaxis $\Delta r_{\|} \sim 2 \lambda / N A^{2} \sim 750 \mathrm{~nm}$. The maximum laser power at the output of the objective is 8.3 $\mathrm{mW}$, corresponding to a power density $\sim 30 \mathrm{~mW} / \mu \mathrm{m}^{2}$, having assumed a diffraction-limited spot area $A=\pi\left(\Delta r_{\perp}\right)^{2} \approx 0.28 \mu \mathrm{m}^{2}{ }^{49}$ After being reflected by an edge filter, the beam is focused by the objective into the glass microcell containing the particle dispersion. The microcell is prepared by depositing a drop $(10 \mu \mathrm{L})$ of solution on a microscope glass slide, on which a $25 \mu \mathrm{m}$ thick, $1 \mathrm{~cm}$ internal radius bi-adhesive ring was pre-attached. A $20 \times 20 \mathrm{~mm}^{2}$ cover slip covers the cell, that is finally sealed on the sides with nail polish. Sealed microcells allow us to prevent water evaporation and carry out analysis on liquid dispersions for several days. 


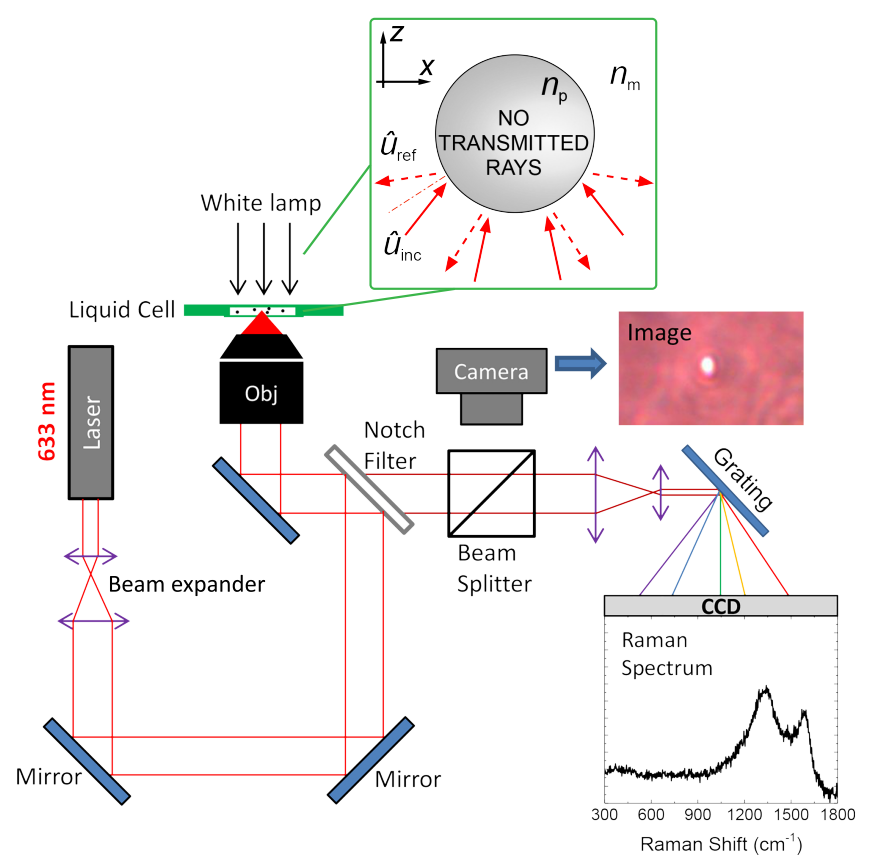

Figure 1: Schematic of the Raman Tweezers setup. The green inset shows the sketch of the ray optics model used to calculate optical forces. $\hat{u}_{\text {inc }}$ and $\hat{u}_{\text {ref }}$ are the directions of the incident and reflected beams, respectively, while $n_{\mathrm{p}}$ and $n_{\mathrm{m}}$ are the refractive index of the particle and of the immersion medium, respectively. See text for a description of the apparatus.

The same objective is used to collect the radiation scattered by the optically trapped particles. The elastic component of the scattering is cut out by the edge filter, which transmits only the Raman component to the spectrometer. The latter is a Triax 190 (Horiba) equipped with a 1200 lines $/ \mathrm{mm}$ grating giving a spectral resolution of $8 \mathrm{~cm}^{-1}$, and coupled to a silicon Peltier-cooled CCD camera. On the way to the spectrometer, a beam splitter redirects part of the light from the white light lamp to a second CMOS camera used for particle visualization and seizing. For each particle, a Raman spectrum is acquired and a reference spectrum (without any trapped particle) is subtracted in order to remove the signal from the glass slide and the medium (water, immersion oil). In order to retrieve the particle's size, an image is acquired after ensuring optimal focusing conditions. Alternatively, when we record a movie of the trapping process, we retrieve the size information from the frames in which the particle is best focused. The optical images are calibrated with a $10 \mu \mathrm{m}$ step ruler. Particles with diameter $d$ larger than the diffraction limit ( $d \gtrsim 500 \mathrm{~nm}$ for visible light using a $N A=1.3$ objective) 
can be measured. Particles smaller than $\sim 500 \mathrm{~nm}$ provide the same diffraction-limited image, making impossible to retrieve the real particle size and/or to determine if one or more particles are actually trapped. ${ }^{50}$ When dealing with sub-diffraction particles, some information on the particles effective volume can be still retrieved, based on the fact that the Raman intensity is expected to scale as the particle volume. In this case a calibration curve of the Raman signal vs particle radius on model particles is needed. ${ }^{46}$

Optical forces. When a particle is illuminated by a tightly focused laser beam, the exchange of linear momentum between the ray and the particle generates an optical force $\vec{F}=\Delta \vec{P} / \Delta t$, where $\Delta \vec{P}=$ $\vec{P}_{\text {inc }}-\vec{P}_{\text {ref }}$ is the exchanged momentum, calculated as the difference between the incident and reflected momentum $\left(\vec{P}_{\text {inc }}\right.$ and $\vec{P}_{\text {ref }}$, respectively). Such a force can either push the particle or pull it into the focal spot. ${ }^{51}$ Stable $3 \mathrm{D}$ optical trapping at position $\vec{r}_{\mathrm{eq}}$ very near to the laser focus, is obtained if the total optical force $\vec{F}_{\text {tot }}(\vec{r})$ points towards the focal spot position $\left(\vec{r}_{\text {eq }}\right)$ for any small displacement $\Delta \vec{r}=\vec{r}-\vec{r}_{\mathrm{eq}}$ from $\vec{r}_{\mathrm{eq}}$. In this case $\vec{r}_{\mathrm{eq}}$, represents a point of stable equilibrium and, for small displacements, the restoring force is well approximated by Hook's law $\vec{F}_{\text {tot }}=-\overleftrightarrow{k} \cdot \Delta \vec{r}$. Here $\overleftrightarrow{k}$ is a three-dimensional tensor describing the direction-dependent stiffness of the trap. In the reference system in which the $z$ axis coincides with the beam propagation direction and $x y$ is the plane perpendicular to $z, \overleftrightarrow{k}$ is represented by a diagonal matrix with eigenvalues $k_{x}, k_{y}, k_{z}$, and $k_{x}=k_{y}$. A rigorous calculation of optical forces within the electromagnetic theory, requires a full description of the light-matter interaction, which can result in a quite complex, time-consuming and unwieldy procedure. For this reason, some approximations have been developed to calculate optical forces in different regimes, depending on the particle size. In the geometrical optics approximation, valid for particles size larger than the wavelength of the trapping beam, the incoming optical field can be considered as a collection of light rays, each of them carrying a portion of the total optical power and linear momentum ${ }^{52}$. When a ray impinges on a particle, it is partly transmitted and partly reflected 
by the particle surface. The change of momentum of each light ray induces a force on the particle (Newton's third law). The total optical force that a beam exerts on a particle is the sum of the forces generated by each constituting ray. Non-absorbing dielectric particles, featuring a real refractive index $n_{\mathrm{p}}$, can be stably trapped in $3 \mathrm{D}$ whenever their refractive index is larger than that of the immersion medium $n_{\mathrm{m}}$, i.e. $n_{\mathrm{p}}>n_{\mathrm{m}}$. This happens for the vast majority of plastics as, for example, polystyrene $\left(n_{\mathrm{p}}=1.59\right)$, polypropylene $\left(n_{\mathrm{p}}=1.49\right)$, polyethylene $\left(n_{\mathrm{p}}=1.51\right)$ when immersed in water $\left(n_{\mathrm{m}}=1.33\right) .{ }^{46}$ Absorbing particles are characterized by a complex refractive index $n_{p}=n_{r}+$ $i n_{k}$, with non-zero imaginary part $n_{k}$. In this case the transmitted ray is totally absorbed by the particle, resulting in a large radiation pressure that generally pushes the particle out of the optical trap. Here optical forces are calculated on micrometric particles by using an open source toolbox written in MATLAB - Optical Tweezers in Geometrical Optics (OTGO), well tested on dielectric particles. ${ }^{53}$ The routines have been modified for optical forces calculation on spherical and ellipsoidal particles, in which the Fresnel coefficients are evaluated in the case of materials with complex refractive index $n_{p}$ (see Supplementary Note 1 for further details). The laser beam is assumed to propagate along the positive $z$ direction, be composed by an appropriate grid of rays having 40 azimuthal and 100 radial divisions and focused to the origin of the reference axes through an objective with high numerical aperture $(N A=1.3)$. Spherical particles are considered, immersed in water (refractive index $\left.n_{\mathrm{m}}=1.33\right)$. Particles are assumed to have different diameters $(d=1,2$ and $4 \mu \mathrm{m})$ and made of either amorphous carbon (refractive index $\left.n_{\mathrm{p}}=2.4+0.6 i\right)$ or graphite $\left(n_{\mathrm{p}}=2.6+\right.$ $1.0 i),{ }^{54}$ similarly to the analyzed TWRPs in this work. With such parameters we calculate the optical force $\vec{F}_{\text {tot }}$ on a particle placed at position $\vec{r}$. Due to the cylindrical symmetry of the system around the light propagation axis, $z$, the $x$ and $y$ directions are equivalent, allowing us to consider only the displacements along the $x$ - and $z$-axis for a full characterization of the optical force as a function of the position $\vec{r}(x, z)$. When the particle is displaced along the propagation axis only, i.e., $\vec{r}=(0, z)$, the force has a purely longitudinal component, $\vec{F}_{\|}(z)$, directed along the axis of the beam, that is 
$\vec{F}_{\text {tot }}(0, z)=\vec{F}_{\|}(z)$. When we displace the particle also along $x$, i.e., $\vec{r}=(x, z)$, the force includes also a transversal component $\vec{F}_{\perp}(x, z)$, i.e., a component directed perpendicularly to the axis of the beam, and therefore $\vec{F}_{\text {tot }}(x, 0, z)=\vec{F}_{\|}(x, z)+\vec{F}_{\perp}(x, z)$. For symmetry reasons, $\vec{F}_{\perp}(x, z)$ is directed along the $x$-axis and the component of the force along the $y$-axis is zero. Here we study the force $\vec{F}_{\|}(z)$ for purely longitudinal displacements $\vec{r}=(0, z)$, and $\vec{F}_{\perp}(x)$ for purely transversal displacements $\vec{r}=(x, 0)$, then we fix a value for $z=z_{0}$ and we study $\vec{F}_{\perp}\left(x, z=z_{0}\right)$. From the behavior of $\vec{F}_{\|}(z)$ and $\vec{F}_{\perp}\left(x, z=z_{0}\right)$ it is possible to predict if the particle is optically trapped or not in $3 \mathrm{D}$. In order to have transversal trapping, the force $\vec{F}_{\perp}\left(x, z=z_{0}\right)$ should become zero for some value $x=x_{\text {eq }}$, i.e., $\vec{F}_{\perp}\left(x_{\text {eq }}, z=z_{0}\right)=0$ when the center of the particle is in $x_{\text {eq }}$. If we displace the particle from $x_{\text {eq }}$ a little bit, the force $\vec{F}_{\perp}\left(x, z=z_{0}\right)$ should be directed opposite to the displacement and point towards $x_{\mathrm{eq}}$, to restore the particle to its equilibrium position. So, when $x>x_{\mathrm{eq}}$, $\vec{F}_{\perp}\left(x, z=z_{0}\right)<0$ and when $x<x_{\text {eq }}$, the force should be positive: $\vec{F}_{\perp}\left(x, z=z_{0}\right)>0$.

\section{Optical trapping of car tires micro- and nano-particles}

Dimensional analysis of mechanically grinded TPs has been carried out with an optical microscope. Figure $2 \mathrm{a}, \mathrm{b}$ shows representative pictures acquired at different magnifications (a: $50 \mathrm{X}$ objective, $\mathrm{b}$ : $10 \mathrm{X}$ objective). The TP dust is opaque, yielding a large contrast in the images. Dimensional analysis carried out on more than 2000 particles using routines written in Mathematica. It highlights a broad distribution of sizes (Figure 2c), ranging from microns to tens of microns. The size distribution rapidly decreases with the diameter (note the loglog scale). A large number of sub-micrometric and micrometric particles (up to $5 \mu \mathrm{m}$ diameter) is observed, compared to a much smaller number of TPs with diameter $>20 \mu \mathrm{m}$. Even if the distribution is likely dictated by the production method, it shows the capacity of weathered car tires to be fragmented into micrometric and even sub-micrometric particles by friction on stones of proper roughness. A variety of shapes is also observed, with circular and very elongated structures, as reported in Figure $2 \mathrm{~d}$ where we display a histogram of the 
eccentricity, $e=\sqrt{1-(b / a)^{2}}$, of the particles shown in Figure 2a. Here $a$ and $b$ are the semiaxes of the best fitted ellipse.
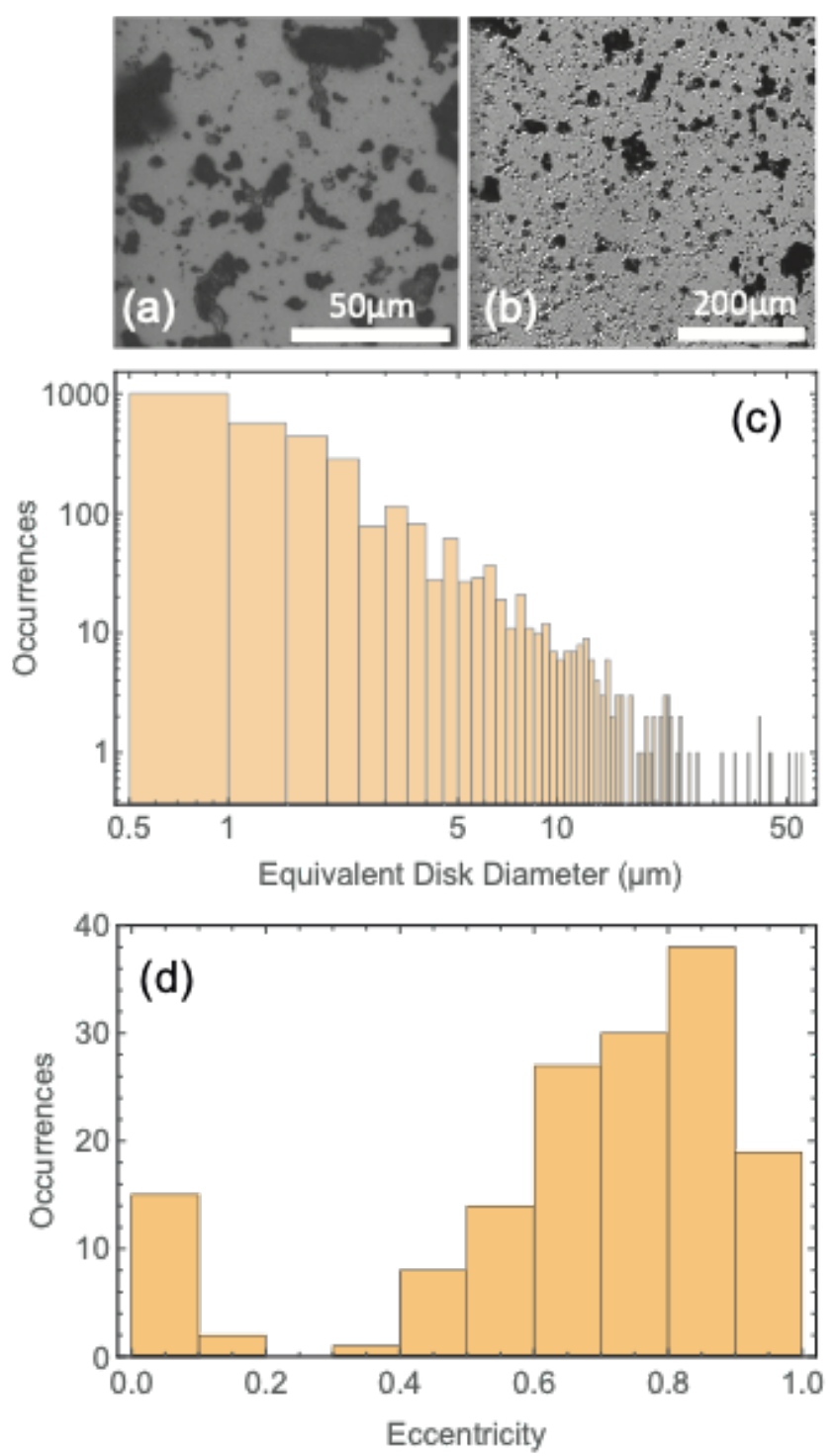

Figure 2: Optical images and statistical analysis of mechanically grinded TPs. (a-b) Microscopy images are acquired at different magnifications (a: 50X objective; b: 10X objective). (c) Particles' diameter distribution shown in loglog scale. (d) Plot of the distribution of the eccentricity of the particles displayed in (a). 


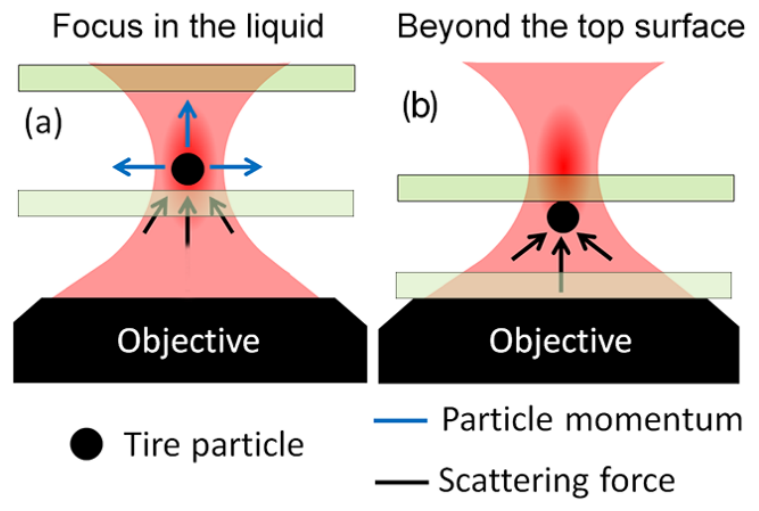

Figure 3: Optical trapping in different focusing conditions. (a) Focus into the liquid: particles are pushed out of the trap. (b) Focus above the top glass cell surface: stable 2D trapping is obtained by pushing the particle against the cell wall.

Optical trapping experiments have been carried out on TPs dispersions in distilled-water, sealed in a 25- $\mu \mathrm{m}$-thick glass fluidic cell. Different focusing conditions have been explored, from inside the liquid (Figure 3a), that is the most conventional configuration for optical trapping, to a configuration in which the beam focus is positioned beyond the top surface of the glass fluidic cell (Figure $3 b$ ). When the beam is focused inside the liquid, we observe that it is not possible to stably trap TPs in 3D. As shown in Supplementary Movie S1, the optical force is such that any particle intercepting the laser beam is pushed away along the light propagation direction, thus escaping from the focal region. In addition, if one tries to drive a particle into the focal region, e.g. by moving the sample with the piezo-stage, the particle is ejected out. The strong radiation pressure can, however, be turned to our advantage by pushing the particles against the top glass cell surface (Supplementary Movie S2) and obtaining some stable trapping. When this is done, the particle can be translated with respect to other floating or precipitated particles. Experimentally, we achieve 2D trapping by focusing the laser spot beyond the top glass cell surface (Figure $3 b$ ). Under such conditions, the particle remains confined in the "defocused" laser beam region (see below for more details), while the reaction of the glass surface prevents it to escape in the vertical direction. This trapping scheme is generally called $2 \mathrm{D}$ optical trapping, since particles are optically trapped in the bidimensional plane orthogonal to the laser beam 
and mechanically in the axial direction. Asymmetric particles start rotating when trapped (Supplementary Movie S2). ${ }^{53,54}$

These phenomena become clearer if we calculate the optical forces acting on strongly absorbing particles. Here we consider model spherical carbon particles and make calculations in the ray optics approximation. Figure $4 \mathrm{a}$ sketches the light rays and the associated momenta impinging on an absorbing particle when the latter is immersed in liquid, and located far from the glass cell surface, with its center $\left(\vec{C}_{0}\right)$ slightly displaced from the laser beam focus position $\left(\vec{r}_{0}\right)$. Figure $4(\mathrm{~b}, \mathrm{c})$ and Figure $4\left(\mathrm{~d}\right.$, e) show, respectively, the transversal, $\vec{F}_{\perp}(x)$, and longitudinal component, $\vec{F}_{\|}(z)$, of the total optical force acting on the particle as a function of the particle displacement in the transverse, $\mathrm{x}$, and longitudinal, z, directions. Calculations are reported for particles with different diameters $(d=$ 1, 2 and $4 \mu \mathrm{m})$, made of amorphous carbon [Figure 4(b, d)], and graphite [Figure 4(c, e)]. 

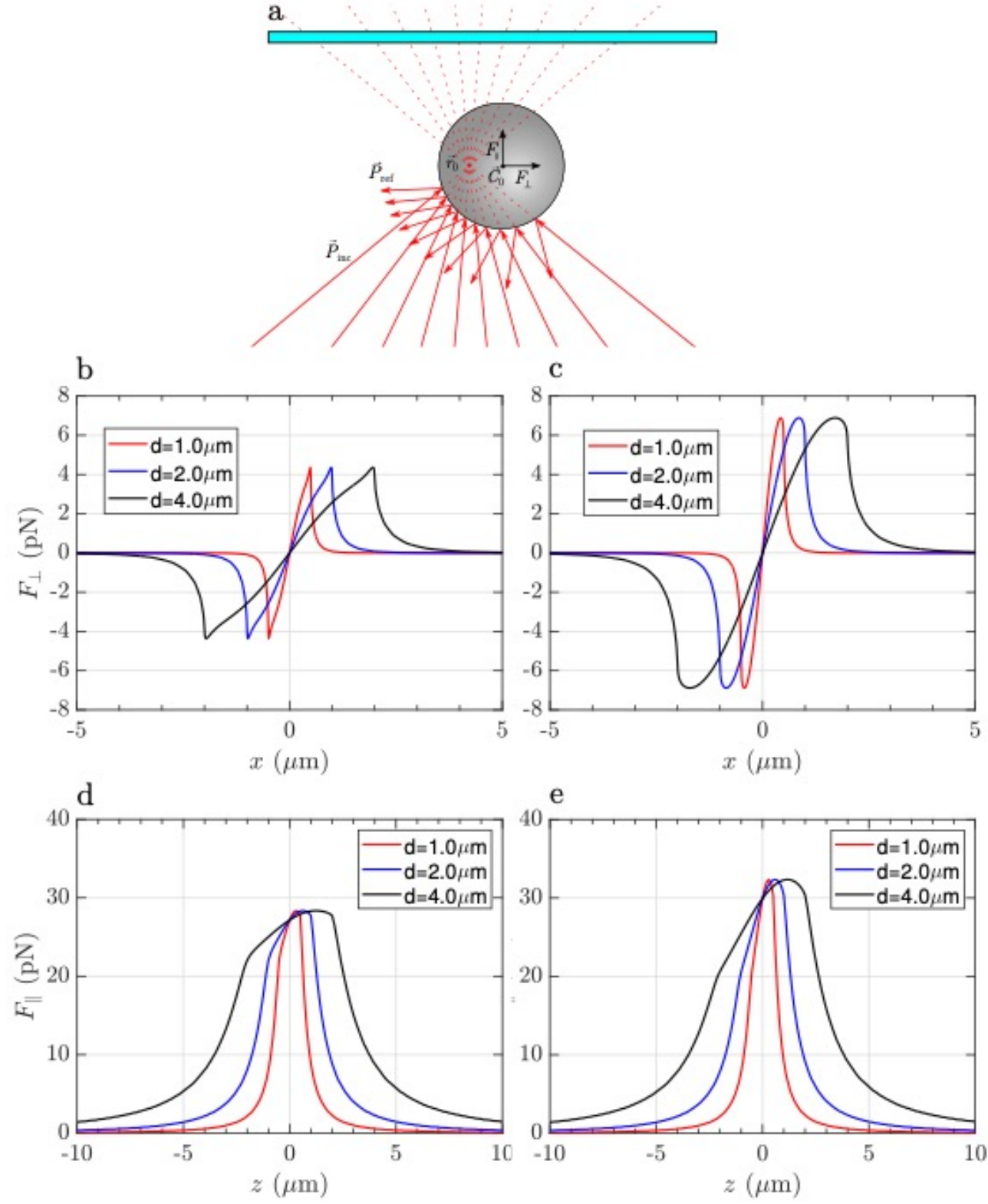

Figure 4: Optical forces acting on carbon microparticles in liquid. (a) Schematic representation of the rays scattered by an absorbing particle when the laser focus position $\vec{r}_{0}$ is in the liquid and far from the glass slide surface (cyan rectangle). The particle's center position is indicated with $\vec{C}_{0} . \vec{P}_{\text {inc }}$ and $\vec{P}_{\text {ref }}$, represented by red arrows, are the incident and reflected linear momenta associated to each light ray. The red dashed lines represent the continuation of the impinging rays, and are a guide to eye to the position of the beam focus. The directions of $\vec{F}_{\|}$and $\vec{F}_{\perp}$ are represented by black arrows originating from the particle center $\vec{C}_{0}$. Transversal (b, c) and longitudinal (d, e) components of the optical force calculated on amorphous carbon (b, d) and graphitic particles (c, e). We consider particles with diameter $d=1 \mu \mathrm{m}$ (red), $2 \mu \mathrm{m}$ (blue), and $4 \mu \mathrm{m}$ (black). 
Figure $4(b, c)$ provides information for what concerns the in-plane trapping. We note that the transversal force vanishes in the focus, i.e. $F_{\perp}(x)=0$ for $x=0$, and acts in the same direction of the displacement, i.e. it is positive when the particle displacement is positive, and vice-versa. This implies that the laser focus is an instable equilibrium position, and that the particle will be ejected once it is slightly displaced off center. The same effect is predicted for all sizes and materials, and correctly describes the experimental observations according to which the particle is ejected from the beam center when we try to pull it into it (Supplementary Movie 1, 00:05 to 00:07 and, in slow motion, 00:21 to 00:26). Due to the higher absorption of graphitic particles, $\operatorname{Im}\left(n_{\mathrm{p}}\right)=1.5$, with respect to the amorphous carbon ones, $\operatorname{Im}\left(n_{\mathrm{p}}\right)=0.6$, the optical force acting on the former is expected to be larger. Regarding the trapping in the axial direction, we note that $F_{\|}(z)$ is positive for any position $z$ [Figure $4(\mathrm{~b}, \mathrm{c})]$, i.e. the longitudinal force is always directed along the beam propagation direction, with a maximum when the particle passes through the focus of the beam $(z=0)$. The particle is expected to be pushed along the beam direction, as observed experimentally (Supplementary Movie 1, 0:00 to 0:03 and, in slow motion, 00:15 to 00:20). In conclusion, no stable $3 \mathrm{D}$ trapping is possible for carbon particles in liquid using $633 \mathrm{~nm}$ light, due to absorption and the consequent large radiation pressure. Pushing and ejection from the laser focus region is predicted and experimentally observed for any position of the beam focus, $\vec{r}_{0}$, inside the liquid.

In Figure 5(a) we show the case of a laser beam focused in liquid, close to the top surface of the glass microcell (the distance of $\vec{r}_{0}$ from the surfce is smaller than the radius of the particle). Looking at the trend of the transversal force, $F_{\perp}(x)$, in Figure $5(\mathrm{~b}, \mathrm{c})$, ejection from the central position, $x=0$, is expected, again, for all particles' sizes and materials. It is noteworthy that, compared to the previous case, now there are several equilibrium positions along the transverse direction, i.e. $\mathrm{x}_{\mathrm{n}}$ for which $F_{\perp}\left(x_{n}\right)=0$. The point $\mathrm{x}_{0}=0$ is unstable, because a displacement determines a force of the same sign. However, the off-center equilibrium positions, $x_{ \pm 1}$, highlighted by red arrows in Figure 5(b, c), are 
potentially stable because the force corresponding to a displacement is opposite in sign, and so it is in the right direction for restoring the particle to the equilibrium.
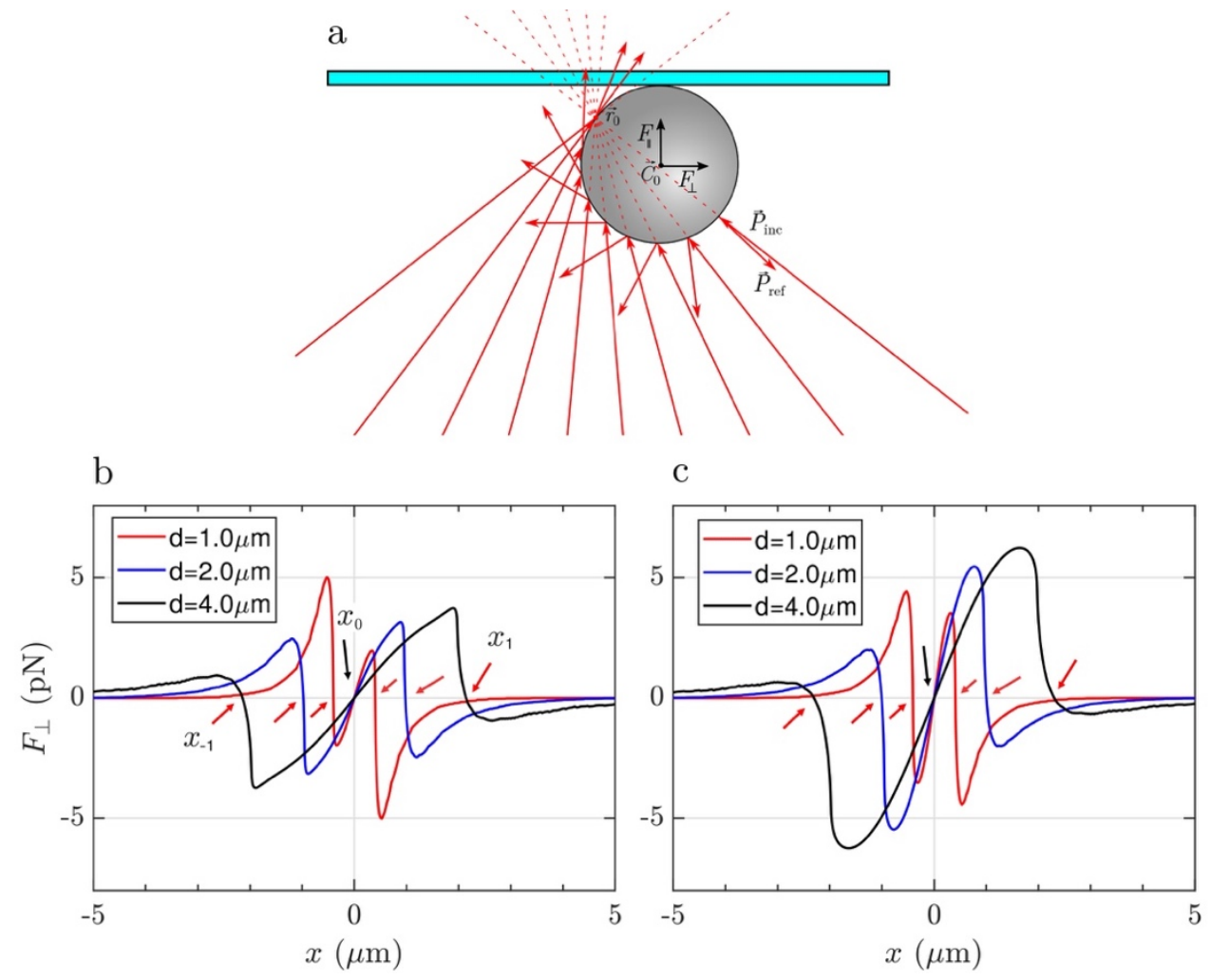

Figure 5: Optical forces acting on carbon microparticles close to the microcell surface. (a)

Schematic of the scattered rays impinging on an absorbing particle when the laser focus position, $\overrightarrow{r_{0}}$, is very close to the top glass surface (cyan rectangle), beyond the particle center $\overrightarrow{C_{0}} \cdot \vec{P}_{\text {inc }}$ and $\vec{P}_{\text {ref }}$, represented by red arrows, are the incident and reflected linear momenta associated to each light ray. The red dashed lines represent the continuation of the impinging rays, and are a guide to eye to the position of the beam focus. The directions of $F_{\|}$and $F_{\perp}$ are represented by black arrows. Transversal component of the optical force $F_{\perp}(x)$ calculated on amorphous carbon (b) and graphite (c) particles for different diameters $d=1 \mu \mathrm{m}$ (red), $2 \mu \mathrm{m}$ (blue), and $4 \mu \mathrm{m}$ (black). The arrows highlight stable (red) and unstable (black) equilibrium positions where the force vanishes. 
Because of the cylindrical symmetry, this corresponds to a circumference of equilibrium points. The particle is potentially trapped in the transversal direction and free to move along the circumference of radius $x_{ \pm 1}$. The effective stability of the off-center equilibrium points depends on how stiff the trap is in that point: if it is too weak, the particle can escape easily because of Brownian fluctuations. For what concerns the longitudinal trapping, also in this case the values of $F_{\|}(z)$ are always positive due to the prevailing radiation pressure. In this configuration the particle is pushed against the cell surface and, due to the reaction force exerted by the surface, the net force acting on it along the $z$ direction will be zero. In conclusion, focusing the light very close to the glass surface, leads to pushing of the particle against the top glass surface, but the particle will likely be trapped off-center or escape from the sides.

Finally, if we focus the laser beyond the surface of the glass slide (Figure 6a), the rays will impinge on the particle surface with different angles compared to the previous two cases, producing a linear momentum exchange which results in a Hook-like restoring transversal force, $F_{\perp}(x)=-k_{x} x$, for small displacements of particle along the $x$ direction, as illustrated in Figure $6(\mathrm{~b}, \mathrm{c})$. This force recalls the particle towards the equilibrium position, $x=0$, i.e. towards the optical axis, whenever it is slightly displaced in the transversal direction, providing stable 2D optical trapping. Differently from the two other cases, we note that the maximum expected force strongly depends on the particle size. This is likely due to the fact that the laser beam at the particle's center position is highly defocused. As a consequence, a bigger particle will intercept a larger number of rays, being subject to a more intense momentum exchange and, finally, to a larger force. As in the previous configurations, the longitudinal force (not shown) is always positive and will push the particle against the glass surface but, once the particle is in contact with this latter, the mechanical constraint reaction of the surface will provide stable trapping also in the axial dimension. Focusing the laser above the top surface, it is therefore possible to trap, analyze and even manipulate absorbing particles by pushing them on the glass surface, as shown in Supplementary Movie S2. 

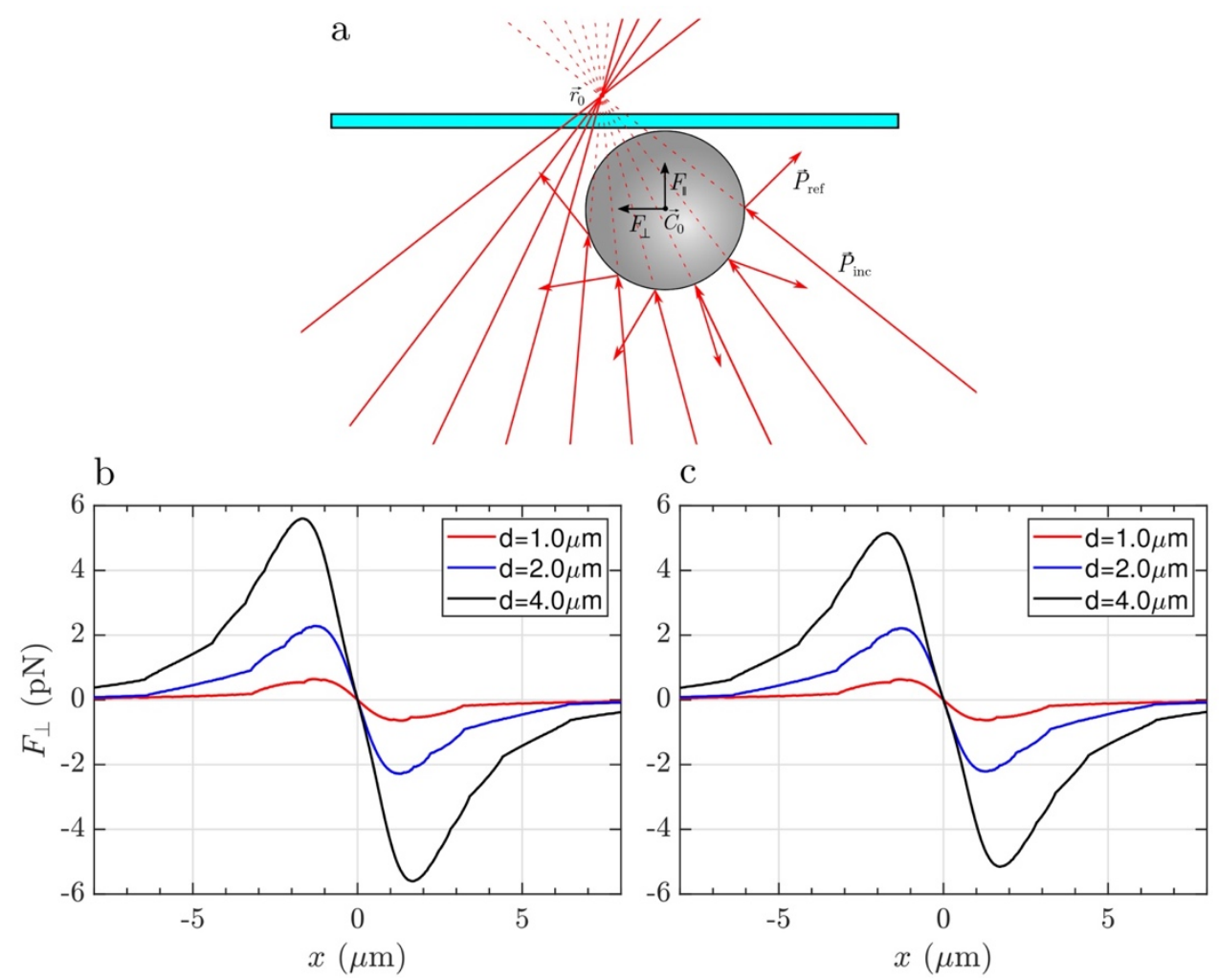

Figure 6: Optical forces acting on carbon microparticles when focusing beyond the glass cell

surface. (a) Schematic representation of the scattered rays by an absorbing particle constrained vertically by the top of the cover glass (cyan rectangle). The laser focus $\overrightarrow{r_{0}}$ is positioned beyond the first surface of the cover glass. $\vec{P}_{\text {inc }}$ and $\vec{P}_{\text {ref }}$ are the incident and reflected linear momenta, respectively, and are represented by red arrows. Red dashed lines represent the continuation of the impinging rays in order to show the position of $\overrightarrow{r_{0}}$, represented by a red dot. The direction of $F_{\|}$and $F_{\perp}$ is represented by black arrows. Transversal component of the optical force $F_{\perp}$ calculated on of amorphous carbon (b) and graphite particles (c) having dimeter $d=1$ (red), 2 (blue), and 3 (black) $\mu \mathrm{m}$.

Two other effects are observed when trying to trap carbon particles. Firstly, when full laser power is focused onto aggregates of TPs in liquid (ca. $8.3 \mathrm{~mW}$ on an area of $0.36 \mu \mathrm{m}^{2}$ ), the generated optical forces are sufficient to disintegrate the aggregate into its smaller constituents (Supplementary Movie S3) and scatter them all around. Secondly, when the laser is tightly focused on individual TP particles, light absorption and local heating yields to micro-cavitation and bubbles generation (Supplementary 
Movie S4, sec. 00:00 to 00:09). The thermal gradients generated by the local laser-induced heating generate convective fluxes in the liquid that, in turn, activate thermophoretic effects resulting in the drag of particles towards the laser spot where they stick (Supplementary Movie S4, sec. 00:10 to 00:22). A similar effect, called thermophoretic trapping, is generated when irradiating plasmonic metal nanoparticles in liquid. ${ }^{55,56}$ Notably, local sample heating is specific to strongly absorbing objects, like carbonaceous particles. No bubbling or thermal damage was observed on conventional polymers or mineral sediments using even higher laser powers. ${ }^{46}$

We can conclude this section saying that, in order to trap individual TRWPs and perform spectroscopic analysis of individual particles, a viable way is to push the particle with full laser power towards the top surface of the liquid cell, being careful not to directly focus the laser on the particle. This ensures that, whenever the particle reaches the top cell surface it remains confined in the focal region and, because of the smaller local density of power, it does not heat up significantly, nor generate bubbles or trigger thermophoretic dragging of other particles. We observed that most of the time the particle would stick to the glass substrate and stay there even with the laser turned off.

\section{Raman spectroscopy of optical trapped TRWP micro- and nano-particles}

First experiments have been carried out on mechanically grinded TRWPs in distilled water, after being stably trapped. Figure 7(a-e) shows the optical images of particles ranging from $600 \mathrm{~nm}$ to 4.6 $\mu \mathrm{m}$ in diameter, together with their Raman spectra in Figure 7(f). Spectra of the particles Figure 7 (ad) were acquired with a defocused laser beam, in order to achieve 2D trapping. The spectrum of the particle in Figure 7 (e) was measured with total power focused just above the glass surface. On all TPs, we observe a Raman spectrum typical of carbon black (amorphous carbon), with bands at 1320

$\mathrm{cm}^{-1}$ ( $\mathrm{D}$ band) and $1580 \mathrm{~cm}^{-1}$ (G band). The $\mathrm{G}$ band is related to the bond stretching of all pairs of $s p^{2}$ bonded atoms in rings and chains, and corresponds to a degenerate in-plane $E_{2 g}$ optical mode at the center of the Brillouin zone. The $\mathrm{D}$ band is observed when there is a symmetry breaking of the $s p^{2}$ 
hexagonal lattices, involving a defect-induced electron-hole "inter-valley" double resonance process that activates a Transverse Optical phonon close to the Brillouin zone boundary K point. ${ }^{57}$

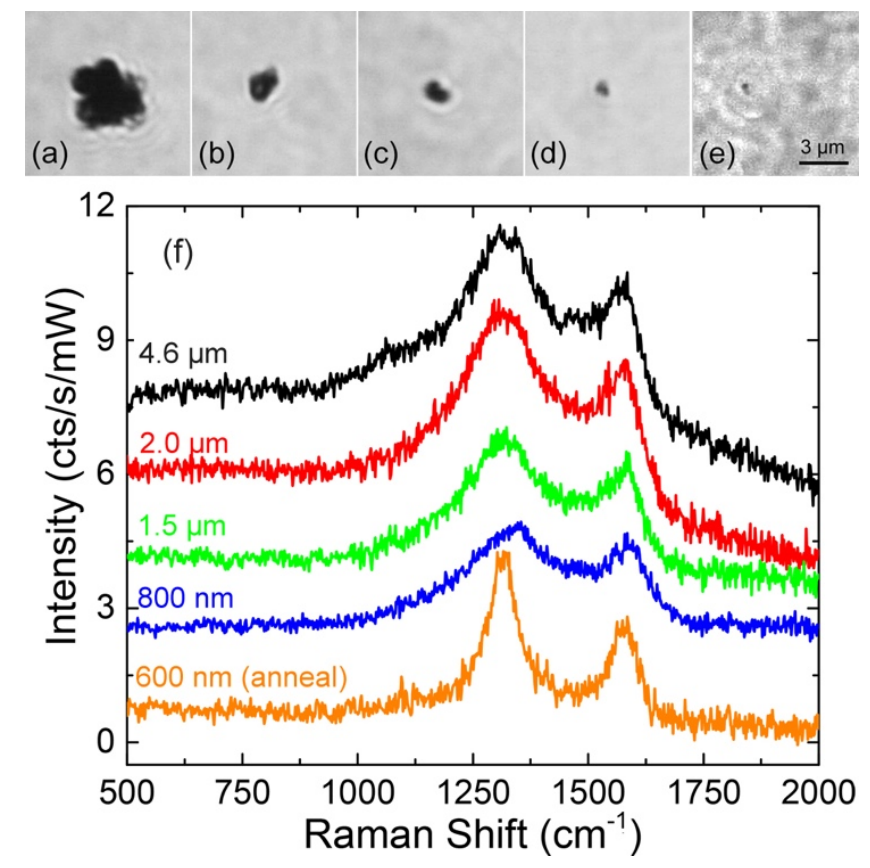

Figure 7: Optical images (a-e) and Raman spectra (f) of 2D-trapped TPs in distilled water. Particles sizes are: (a) of $4.6 \mu \mathrm{m}$, (b) $2.3 \mu \mathrm{m}$, (c) $1.5 \mu \mathrm{m}$ and (d) $850 \mathrm{~nm}$, (e) $600 \mathrm{~nm}$. Acquisition time 60 s for two acquisitions. Laser power out of the objective: $8.3 \mathrm{~mW}$. Raman spectra are acquired with defocused laser for particles (a-d) and with total power focused just above the glass surface (e). All spectra are offset for clarity.

The intensity of the bands is not very sensitive to the size of the particle. This is due to two reasons: first, because the carbon particles are very absorptive and only a thin layer of carbon is effectively irradiated; second, because in 2D trapping conditions the beam is defocused. Since for larger particles a stronger defocusing is needed to achieve stable trapping, a greater loss of Raman signal is expected, justifying why the signal from larger particles is similar to that from smaller ones. The smallest particle we trapped (Figure 7e) was elongated with a size of $600 \times 800 \mathrm{~nm}$, comparable to the laser spot size. The particle upon pushing stably stuck onto the glass cell. Focusing maximum power on it generated a steam bubble for one second and the spectrum acquired just after this annealing presents much sharper bands than those of the other tire particles. The narrowing of the band widths is 
generally associated to an increase of the crystallites sizes. ${ }^{58,59} \mathrm{We}$ can attribute this to the crystallization of the amorphous carbon content in the tire particle induced by the laser heating ${ }^{60}$ this indicates that with such laser power one should be aware that the full power density can alter the TPs structure. Further experiments have been done diluting the tire particles in sea water. Again, we can trap in 2D and identify carbon-rich particles down to dimensions as small as $500 \mathrm{~nm}$ (Supplementary Figure S2), unambiguously differentiating them from sediments (spectra now shown, reported in ref. ${ }^{56}$ ). Regarding the smallest observed particles, since we are at the diffraction limit, we cannot exclude that the actual size is less than $500 \mathrm{~nm}$.

These results prove that, on the one side, abrasion of TPs on finely textured stones leads to the formation of nanoscale particles and, on the other hand, that at $633 \mathrm{~nm}$ RTs can be used for analysis of such nanoplastics, in time scales of one to two minutes. Some other interesting features have been observed. Micrometric scale TPs with clear zones decorated by black spots (Figure 8a) have been found, associated with a Raman spectrum (Figure 8d, blue line) characterized by the simultaneous fingerprints of carbon black and $\mathrm{TiO}_{2}$ in the rutile phase (doublet at 443 and $605 \mathrm{~cm}^{-}$ $\left.{ }^{1}\right){ }^{29}$ This suggests the occurrence of hetero-aggregates containing the mineral mixed with rubber. Similarly, we find that TPs aggregates characterized by whitish zones at the optical microscope (Figure 8(b, c)) are actually composed of a mixture of carbon black and gypsum. The Raman spectra (Figure 8d, black and red lines) display, in fact, the two intense carbon black bands, together with the peaks of $\mathrm{CaSO}_{4} \cdot 2 \mathrm{H}_{2} \mathrm{O}$ (Calcium sulfate dihydrate) at 425, 490, 615, 665, and $1004 \mathrm{~cm}^{-1}$. ${ }^{61}$ For this latter case a commercial software (Wiley, KnowItAll 2020) has been used for materials assignment. The particle in Figure 8(b) has diameter around $1 \mu \mathrm{m}$, with a black TP component ca. $600 \mathrm{~nm}$ large, i.e. in the nanoscale range. The presence of gypsum in traffic-related PM and TWRPs is well known. ${ }^{20}$ What is interesting here is the capability of RT to detect TPs-mineral aggregates of dimensions down to $1 \mu \mathrm{m}$, composed by sub-components of nanometric scale. The richness of the spectral features, beyond the simple Raman bands of carbon, allows us to make speculations on the actual origin of the detected particle. The fact that the analysis can be accomplished in liquid environment, 
on a single particle level and in minutes, is a further unique advantage of RT with respect to conventional Raman analysis which is limited to ensemble measurements when applied to very dense particles dispersions.

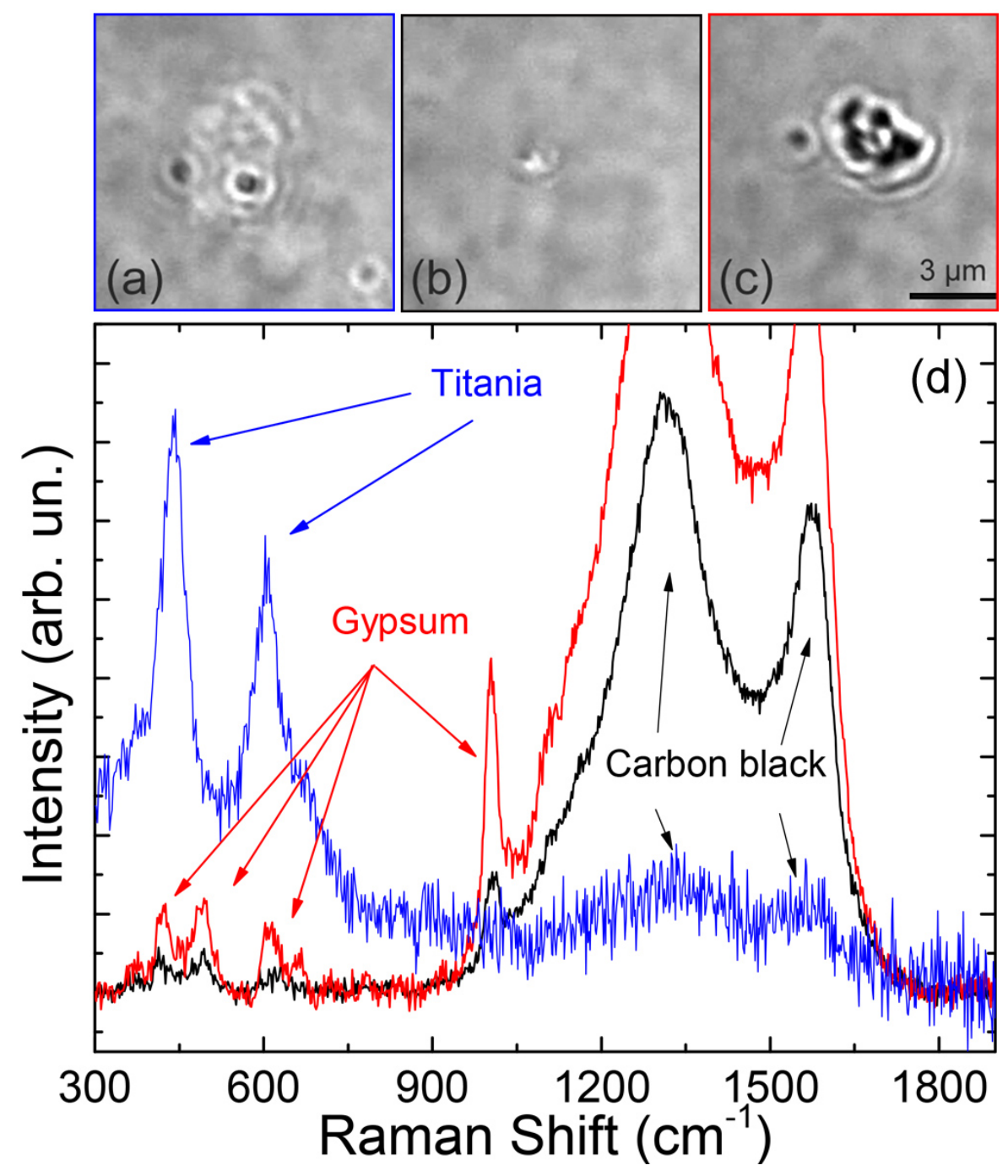

Figure 8: Optical images (a-c) of TPs containing a rubber and a mineral part, together with their Raman spectra (d). The blue line refers to image (a), black line to (b), red line to (c). Integration time $60 \mathrm{~s}, 2$ acquisitions.

Finally, we present the results on TWRPs extracted from a brake test platform. To show the potentialities of the RT technique, we have analyzed 12 particles with dimensions from $4.5 \mu \mathrm{m}$ down to $600 \mathrm{~nm}$. Figure 9 shows the optical images and Raman spectra of representative particles made of $\mathrm{TiO}_{2}$ rutile (Figure 9(a,e) - black and green lines) and carbon black (Figure 9(b,e) - red line). The 
presence of rutile can be attributed to small residues of road dust. ${ }^{62}$ Carbon black is the fingerprint of tire particles, although at this stage we cannot exclude an origin related to exhaust combustion particles. In our ensemble we found 3 rutile particles with dimensions of $1.6 \times 0.5 \mu \mathrm{m}$ (image in Figure 9a, spectrum in Figure 9e - green line), $850 \mathrm{~nm}$ (spectrum in Figure 9e - black line) and $2.2 \mu \mathrm{m}$ (not shown), and 4 carbon black particles with dimensions from $2.4 \mu \mathrm{m}$ (image in Figure 9b, spectrum in Figure $9 \mathrm{e}-$ red line) to $1.1 \mu \mathrm{m}$ (not shown). Particles of smaller dimensions in Figure $9 \mathrm{c}($ size $700 \times 500$ $\mathrm{nm}$ ) and Figure 9d (size $600 \mathrm{~nm}$ ), namely in the nanoscale regime, show even more interesting Raman spectra. Together with the amorphous carbon peaks, we find a single additional band around $665 \mathrm{~cm}^{-}$ ${ }^{1}$ (Figure 9e, cyan line) for the particle in (c), and several further peaks (Figure 9e, blue line) for the particle in (d), suggesting that we are dealing heterogeneous composites. The band at $665 \mathrm{~cm}^{-1}$ is typical of magnetite $\left(\mathrm{Fe}_{3} \mathrm{O}_{4}\right),{ }^{63,64}$ used as abrasive material in brake pads. ${ }^{3,65}$ Consequently, the particle in (c) is likely a brake nanoparticle with a carbon content coming from intrinsic constituents, e.g. burnt lubricant. The complex spectrum of the $600 \mathrm{~nm}$ nanoparticle in (d) has been also observed on a $3.6 \mu \mathrm{m}$ diameter structure, with higher signal-to-noise ratio (inset of Figure 9e, black line). The strong band at $660 \mathrm{~cm}^{-1}$ suggests the presence of some iron oxide species but, this time, it shows up side by side with a peak at $610 \mathrm{~cm}^{-1}$ and other peaks at 300,414 and $500 \mathrm{~cm}^{-1}$. 


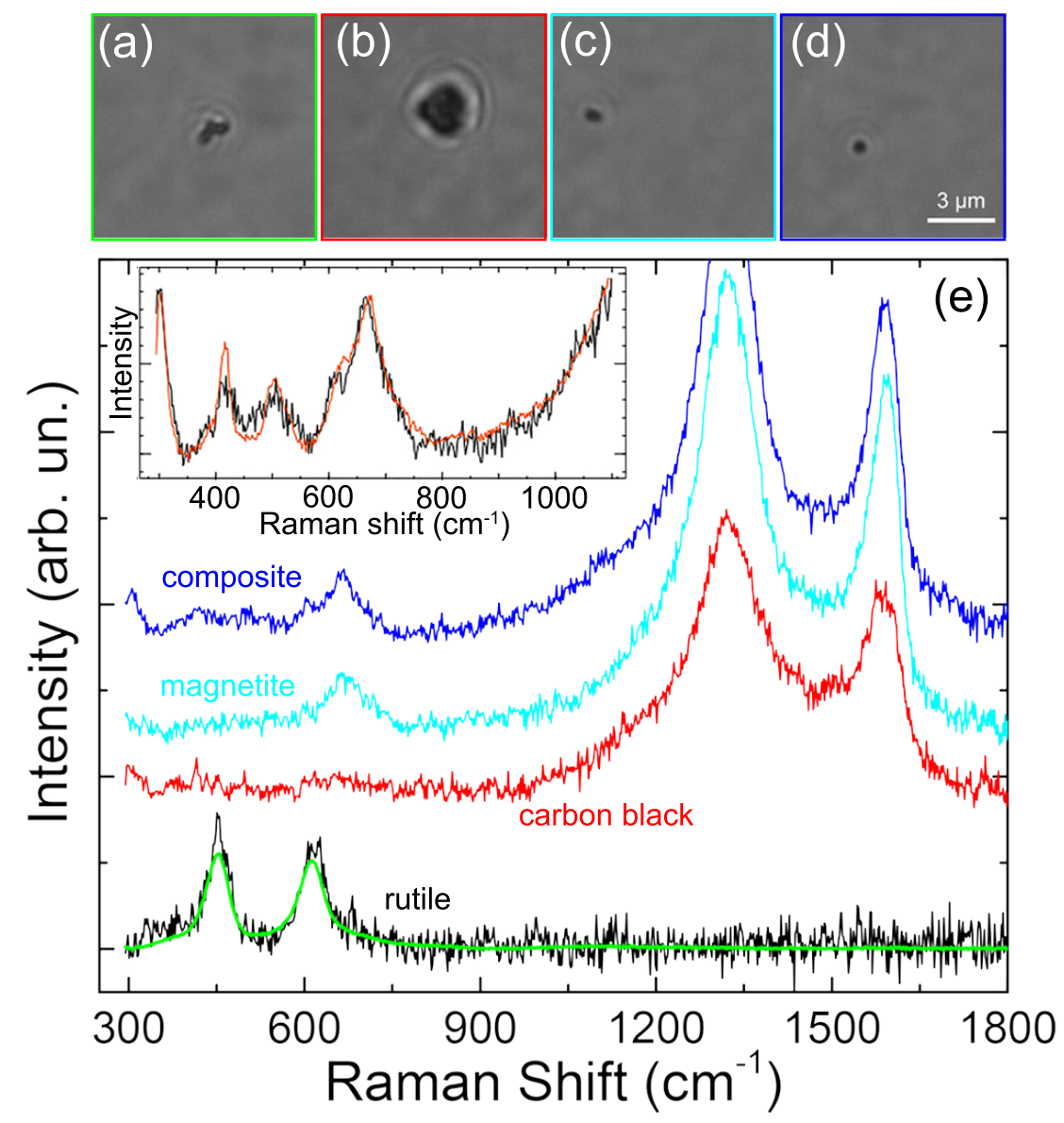

Figure 9: Optical images (a-d) and Raman spectra (e) of trapped particles sampled from a brake test platform. Particles sizes are: (a) $1.6 \times 0.5 \mu \mathrm{m}$, (b) $2.4 \mu \mathrm{m}$, (c) $700 \times 500 \mathrm{~nm}$ and (d) $600 \mathrm{~nm}$. Raman spectra: green line refers to particle (a), red line to particle (b), cyan line to particle (c), blue line to particle (d). The black line refers to a smaller, $850 \mathrm{~nm}$, rutile particle (image not shown). The inset shows a zoom of the spectrum acquired on a $3.6 \mu \mathrm{m}$ composite particle (black line) with a reconstruction (red line) carried out from commercial Raman libraries. Acquisition time: 30s, two acquisitions. All spectra are offset for clarity.

The peaks at $300,410 \mathrm{~cm}^{-1}$ together with the doublet $610-660 \mathrm{~cm}^{-1}$ are software-assigned (Bio-Rad, KnowItAll) to the Raman emission of Red Ochre and Burnt Sienna, ${ }^{66}$ the peak at $500 \mathrm{~cm}^{-1}$ is assigned to lead oxide, the two major bands at 1325 and $1587 \mathrm{~cm}^{-1}$ to carbon black. The red line in the inset of Figure 9e is the software-reconstructed overall spectrum, which fits well with the experiment. Lead oxide is a component of brake friction materials. ${ }^{67}$ Red Ochre and Burnt Sienna are red-colored powders obtained from the intense heating, i.e. calcination, of Limonite and raw Sienna earths, 
minerals present in iron-oxide reach earths that present in Sicily. The simultaneous occurrence of lead oxide, calcinated minerals and carbon black is compatible with the spectrum expected from a heterogeneous structure composed of a fragment of braking pads and road dust particle that has undergone intense heating, e.g. during braking, covered by some lubricant layer (carbon rich).

Based on the experiments shown we can draw some considerations on the information provided by Raman Tweezers in the field of TRWP. RT allows one to analyze individual carbon-rich micro and nanoparticles in water, by isolating them one by one. Indeed, the carbon fingerprint observed on TPs has little specificity, if any, and the actual origin of the particle (tires particle, exhaust particle, lubricant, ...) is almost impossible to be determined, d'emblée, unless some pre-knowledge of the sample is present. On the other hand, based on additional spectral features that can be found in the Raman spectra, some more specific information on the actual composition and origin of the investigated particle can be acquired.

\section{Conclusion}

In conclusion, we have used Raman Tweezers to trap and analyze Tire and Road Wear Particles with dimensions from few microns down to the $500 \mathrm{~nm}$ range (at the diffraction limit of our imaging system). We show both theoretically and experimentally, that the strong radiation pressure observed on such absorbing particles prevents stable trapping in $3 \mathrm{D}$, but that $2 \mathrm{D}$ trapping can be accomplished by pushing the particles against the top surface of the glass cell in which they are contained. Light irradiation can lead to the fragmentation of TPs aggregates into their smaller constituents. Strong light focusing on a single car tire particle generates bubbling and can even induce changes in its crystalline structure. Overheated carbon particles generate thermal gradients inside the water medium, triggering thermophoretic flows of matter, dragging and concentrating particles in the focal region of the laser, and finally leading to a thermophoretic trapping effect. We have observed that particles produced by mechanical grinding of aged tires can reach sizes down to the nanometric range (500nm or below). These particles have been optically trapped in distilled and seawater and analyzed by Raman 
spectroscopy in times of the order of 1 minute. Thanks to the richness of information provided by the Raman spectra, we have been able to identify the presence of hetero-aggregates composed of a mixture of road minerals and rubber. Finally, experiments carried out on particles extracted from a brake test platform have shown the capability of RTs to discriminate among road particulate matter, tire particles, and brake wear particles with dimensions down to ca. $600 \mathrm{~nm}$. Based on these results, we believe that RTs could have a potential application for studying the presence of Tire and Road Wear nanoparticles in water systems, avoiding artifacts related to particles re-aggregation during the drying phase required by other conventional analytical techniques (SEM, microFT-IR, conventional microRaman). Technological efforts are still needed in order to automatize the analysis process and for image and spectral recognition. In this regard, the implementation of artificial intelligence and deep learning routines is foreseen as a necessary step in future research.

\section{Acknowledgments}

This work has been funded by IFREMER through the project MERLIN-MICROPLASTIQUE (agreement No. 17/1212947B). Partial financial contribution from the agreement ASI-INAF n.2018-16-HH.0, project "SPACE Tweezers" and from the MSCA ITN (ETN) project "Active Matter" is also acknowledged.

\section{Statements}

The authors declare no conflict of interest.

\section{Authors Contribution}

R. Gillibert: investigation, methodology, data curation, visualization, writing - original draft; A. Magazzù, A. Callegari: conceptualization, investigation, methodology, software, writing - review \& editing; A. Foti, D. Bronte-Ciriza, M. G. Donato: writing - review \& editing; M. Lamy de La Chapelle, G. Volpe: supervision, writing - review \& editing; O. M. Maragò: funding acquisition, 
supervision, writing - review \& editing; F. Lagarde: Funding acquisition, project administration, writing - review \& editing; P. G. Gucciardi: conceptualization, supervision, funding acquisition, project administration, writing - original draft, writing - review \& editing

\section{References}

1 C. M. S. Ahmed, H. Jiang, J. Y. Chen and Y.-H. Lin, Atmosphere, 2018, 9, 336.

2 A. Mukherjee and M. Agrawal, Environ. Chem. Lett., 2017, 15, 283-309.

3 T. Grigoratos and G. Martini, Environ. Sci. Pollut. Res., 2015, 22, 2491-2504.

4 S. Wagner, T. Hüffer, P. Klöckner, M. Wehrhahn, T. Hofmann and T. Reemtsma, Water Res., 2018, 139, 83-100.

5 S. Wagner and T. Reemtsma, Nat. Nanotechnol., 2019, 14, 300.

6 I. Wagner, Global tire demand - cars and light CVs 2018, https://www.statista.com/statistics/792209/global-tire-demand/, (accessed July 13, 2020).

7 C. Vogelsang, A. L. Lusher, M. E. Dadkhah, I. Sundvor, M. Umar, S. B. Ranneklev, D. Eidsvoll and S. Meland, Microplastics in road dust - characteristics, pathways and measures, Norsk institutt for vannforskning, 2019.

8 K. M. Unice, M. P. Weeber, M. M. Abramson, R. C. D. Reid, J. A. G. van Gils, A. A. Markus, A. D. Vethaak and J. M. Panko, Sci. Total Environ., 2019, 646, 1639-1649.

9 A. Wik and G. Dave, Chemosphere, 2005, 58, 645-651.

10 A. Wik and G. Dave, Chemosphere, 2006, 64, 1777-1784.

11 Miguel A G, Cass G R, Weiss J, and Glovsky M M, Environ. Health Perspect., 1996, 104, 1180 1186.

12 J. M. Horner, Rev. Environ. Health, , DOI:10.1515/REVEH.1996.11.4.175.

13 A. A. Elnazer, S. A. Salman, E. M. Seleem and E. M. Abu El Ella, Int. J. Ecol., 2015, 2015, 1-7.

14M. Huber, A. Welker and B. Helmreich, Sci. Total Environ., 2016, 541, 895-919.

15M. Jenkel, 2019. 
16J. Padovan, N. Prasad, D. Gerrard, S. W. Park and N. Lindsley, Rubber Chem. Technol., 1999, 72, $343-356$.

17 M. L. Kreider, J. M. Panko, B. L. McAtee, L. I. Sweet and B. L. Finley, Sci. Total Environ., 2010, 408, 652-659.

18K. Adachi and Y. Tainosho, Environ. Int., 2004, 30, 1009-1017.

19M. Camatini, G. F. Crosta, T. Dolukhanyan, C. Sung, G. Giuliani, G. M. Corbetta, S. Cencetti and C. Regazzoni, Mater. Charact., 2001, 46, 271-283.

20F. Sommer, V. Dietze, A. Baum, J. Sauer, S. Gilge, C. Maschowski and R. Gieré, Aerosol Air Qual. Res., 2018, 18, 2014-2028.

21 Aatmeeyata and M. Sharma, Sci. Total Environ., 2010, 408, 4563-4568.

22 C. Lassen, S. F. Hansen, K. Magnusson, N. B. Hartmann, P. R. Jensen, T. G. Nielsen and A. Brinch, Microplastics: Occurrence, effects and sources of releases to the environment in Denmark, Danish Environmental Protection Agency, 2015.

23 M. Siegfried, A. A. Koelmans, E. Besseling and C. Kroeze, Water Res., 2017, 127, 249-257.

24C. Schwaferts, R. Niessner, M. Elsner and N. P. Ivleva, TrAC Trends Anal. Chem., 2019, 112, 5265.

25 W. F. Rogge, L. M. Hildemann, M. A. Mazurek, G. R. Cass and B. R. T. Simoneit, Environ. Sci. Technol., 1993, 27, 1892-1904.

26K. M. Unice, M. L. Kreider and J. M. Panko, Int. J. Environ. Res. Public. Health, 2012, 9, 40334055.

27 M. Kovochich, M. Liong, J. A. Parker, S. C. Oh, J. P. Lee, L. Xi, M. L. Kreider and K. M. Unice, Sci. Total Environ., 2021, 757, 144085.

28D. A. Long, The Raman effect: a unified treatment of the theory of Raman scattering by molecules, Wiley, Chichester; New York, 2002.

29I. R. Lewis and H. G. M. Edwards, Eds., Handbook of Raman spectroscopy: from the research laboratory to the process line, Marcel Dekker, New York, 2001. 
30P. G. Gucciardi, S. Trusso, C. Vasi, S. Patanè and M. Allegrini, in Applied Scanning Probe Methods V: Scanning Probe Microscopy Techniques, eds. B. Bhushan, S. Kawata and H. Fuchs, Springer Berlin Heidelberg, Berlin, Heidelberg, 2007, pp. 287-329.

31 A. Foti, F. Barreca, E. Fazio, C. D’Andrea, P. Matteini, O. M. Maragò and P. G. Gucciardi, Beilstein J. Nanotechnol., 2018, 9, 2718-2729.

32 N. Mauser and A. Hartschuh, Chem Soc Rev, 2014, 43, 1248-1262.

33 J. Lee, K. T. Crampton, N. Tallarida and V. A. Apkarian, Nature, 2019, 568, 78-82.

34D. Elkhatib and V. Oyanedel-Craver, Environ. Sci. Technol., 2020, 54, 7037-7049.

35F. Stock, C. Kochleus, B. Bänsch-Baltruschat, N. Brennholt and G. Reifferscheid, TrAC Trends Anal. Chem., 2019, 113, 84-92.

36P. Li, Q. Li, Z. Hao, S. Yu and J. Liu, J. Environ. Sci., 2020, 94, 88-99.

37 M. F. Costa, J. Pinto da Costa and A. C. Duarte, Curr. Opin. Environ. Sci. Health, 2018, 1, 36-40. 38 A. Ashkin, J. M. Dziedzic, J. E. Bjorkholm and S. Chu, Opt. Lett., 1986, 11, 288-290.

39O. M. Maragò, P. H. Jones, P. G. Gucciardi, G. Volpe and A. C. Ferrari, Nat. Nanotechnol., 2013, 8, 807-819.

40 D. V. Petrov, J. Opt. Pure Appl. Opt., 2007, 9, S139.

41 R. Thurn and W. Kiefer, Appl. Spectrosc., 1984, 38, 78-83.

42 O. M. Maragó, F. Bonaccorso, R. Saija, G. Privitera, P. G. Gucciardi, M. A. Iatì, G. Calogero, P.

H. Jones, F. Borghese, P. Denti, V. Nicolosi and A. C. Ferrari, ACS Nano, 2010, 4, 7515-7523.

43 T. Rodgers, S. Shoji, Z. Sekkat and S. Kawata, Phys. Rev. Lett., 2008, 101, 127402.

44E. Messina, L. D’Urso, E. Fazio, C. Satriano, M. G. Donato, C. D’Andrea, O. M. Maragò, P. G. Gucciardi, G. Compagnini and F. Neri, J. Quant. Spectrosc. Radiat. Transf., 2012, 113, 24902498.

45 M. Wu, D. Ling, L. Ling, W. Li and Y. Li, Sci. Rep., 2017, 7, 42930. 
46R. Gillibert, G. Balakrishnan, Q. Deshoules, M. Tardivel, A. Magazzù, M. G. Donato, O. M. Maragò, M. Lamy de La Chapelle, F. Colas, F. Lagarde and P. G. Gucciardi, Environ. Sci. Technol., 2019, 53, 9003-9013.

47C. Schwaferts, V. Sogne, R. Welz, F. Meier, T. Klein, R. Niessner, M. Elsner and N. P. Ivleva, Anal. Chem., , DOI:10.1021/acs.analchem.9b05336.

48 S. Zhang, Y. Sun, B. Liu and R. Li, Sci. Total Environ., 2020, 143504.

49M. Labardi, P. G. Gucciardi and M. Allegrini, Riv. Nuovo Cimento, 2000, 23, 1-35.

50L. Novotny and B. Hecht, Principles of Nano-Optics, Cambridge University Press, 2012.

51 P. H. Jones, O. M. Maragò and G. Volpe, Optical tweezers: Principles and applications, Cambridge University Press, 2015.

52 A. Ashkin, Biophys. J., 1992, 61, 569-582.

53 A. Callegari, M. Mijalkov, A. B. Gököz and G. Volpe, JOSA B, 2015, 32, B11-B19.

54 W. W. Duley, Astrophys. J., 1984, 287, 694.

55G. Baffou, Thermoplasmonics: Heating Metal Nanoparticles Using Light, Cambridge University Press, Cambridge, United Kingdom ; New York, NY, 1 edition., 2017.

56R. Gillibert, F. Colas, M. L. de La Chapelle and P. G. Gucciardi, Plasmonics, , DOI:10.1007/s11468-020-01128-4.

57 A. C. Ferrari and J. Robertson, Phys. Rev. B, 2000, 61, 14095-14107.

58 L. G. Cançado, M. G. da Silva, E. H. M. Ferreira, F. Hof, K. Kampioti, K. Huang, A. Pénicaud, C.

A. Achete, R. B. Capaz and A. Jorio, 2D Mater., 2017, 4, 025039.

59P. Puech, M. Kandara, G. Paredes, L. Moulin, E. Weiss-Hortala, A. Kundu, N. Ratel-Ramond, J.M. Plewa, R. Pellenq and M. Monthioux, C, 2019, 5, 69.

60 J. Ribeiro-Soares, M. E. Oliveros, C. Garin, M. V. David, L. G. P. Martins, C. A. Almeida, E. H. Martins-Ferreira, K. Takai, T. Enoki, R. Magalhães-Paniago, A. Malachias, A. Jorio, B. S. Archanjo, C. A. Achete and L. G. Cançado, Carbon, 2015, 95, 646-652. 
61 A. Rodríguez, K. Eremin, N. Khandekar, J. Stenger, R. Newman, F. Bazeta and M. T. Escohotado, J. Raman Spectrosc., 2010, 41, 1517-1524.

62 A. L. Gallego-Hernández, D. Meza-Figueroa, J. Tanori, M. Acosta-Elías, B. González-Grijalva, J. F. Maldonado-Escalante, S. Rochín-Wong, D. Soto-Puebla, S. Navarro-Espinoza, R. OchoaContreras and M. Pedroza-Montero, Environ. Pollut., 2020, 260, 114006.

63 D. Bersani, P. P. Lottici and A. Montenero, J. Raman Spectrosc., 1999, 30, 355-360.

64P. Panta and C. Bergmann, J. Mater. Sci. Eng., 2015, 5, 3.

65 World Intellectual Property Organization, WO2017170532A1, 2017.

66D. Bikiaris, S. Daniilia, S. Sotiropoulou, O. Katsimbiri, E. Pavlidou, A. P. Moutsatsou and Y. Chryssoulakis, Spectrochim. Acta. A. Mol. Biomol. Spectrosc., 2000, 56, 3-18.

67E. Adamiec, Environ. Geochem. Health, 2017, 39, 1457-1468. 